\title{
Creatine deficiency syndrome
}

INSERM

\section{Source}

INSERM. (1999). Orphanet: an online rare disease and orphan drug data base. Creatine deficiency syndrome. ORPHA:79172

Creatine deficiency syndrome (CDS) comprises a group of inborn errors of creatine metabolism, characterized by a global developmental delay, intellectual disability and associated neurological (seizures, movement disorders, myopathy) and behavioral manifestions. CDS includes two creatine biosynthesis disorders; guanidinoacetate methyltransferase deficiency and L- Arginine: glycine amidinotransferase deficiency, as well as X-linked creatine transporter deficiency. 